\title{
Linear Motion Blur Parameter Estimation in Noisy Images Using Fuzzy Sets and Power Spectrum
}

\author{
Mohsen Ebrahimi Moghaddam and Mansour Jamzad \\ Department of Computer Engineering, Sharif University of Technology, 11365-8639 Tehran, Iran
}

Received 17 July 2005; Revised 11 March 2006; Accepted 15 March 2006

Recommended by Rafael Molina

\begin{abstract}
Motion blur is one of the most common causes of image degradation. Restoration of such images is highly dependent on accurate estimation of motion blur parameters. To estimate these parameters, many algorithms have been proposed. These algorithms are different in their performance, time complexity, precision, and robustness in noisy environments. In this paper, we present a novel algorithm to estimate direction and length of motion blur, using Radon transform and fuzzy set concepts. The most important advantage of this algorithm is its robustness and precision in noisy images. This method was tested on a wide range of different types of standard images that were degraded with different directions (between $0^{\circ}$ and $180^{\circ}$ ) and motion lengths (between 10 and 50 pixels). The results showed that the method works highly satisfactory for SNR $>22 \mathrm{~dB}$ and supports lower SNR compared with other algorithms.
\end{abstract}

Copyright @ 2007 Hindawi Publishing Corporation. All rights reserved.

\section{INTRODUCTION}

The aim of image restoration is to reconstruct or estimate an uncorrupted image by using the degraded version of the same image. One of the most common degradation functions is linear motion blur with additive noise. Equation (1) shows the relationship between the observed image $g(x, y)$ and its uncorrupted version $f(x, y)[1]$ :

$$
g(x, y)=f(x, y) * h(x, y)+n(x, y)
$$

In this equation, $h$ is the blurring function (or point spread function (PSF)), that is, convolved in the original image and $n$ is the additive noise function. According to (1), in order to determine the uncorrupted image, we need to find the blurring function $(h)$ (i.e., blur identification) which is an ill-posed problem. Finding motion blur parameters in none additive noise environments was addressed in [2-4], where these researchers tried to extend their algorithms to noisy images as well. The authors in $[4,5]$ have divided the image into several windows to reduce noise effects and to extend their methods to support noisy images. Linear motion blur identification in noisy images was also addressed using bispectrum in $[3,6]$. This method is not precise enough because theoretically, to remove the noise by using this method, many windows are required, which in practice is impossible. The authors in $[3,6]$ did not specify the lowest SNR that their method can support. A different method was presented for noisy images in [2] where authors used AR (auto regressive) model to present images and have proved the lowest allowed SNR that their method can support. In [7], we presented a method based on mathematical modeling to estimate parameters in noisy images at low SNRs.

In many other research areas, fuzzy concepts have been used to improve the application performance and speed. In the field of image restoration, some researchers have applied fuzzy concepts as well, however, most of these works are in blind restoration. For example, authors in [8] presented a method that incorporated domain knowledge while preserving the flexibility of the scheme. In the most of other papers, only the noise removal methods were presented. In $[9,10]$ a method was presented using fuzzy concepts to remove MF (median filter) side effects such as distortion using an HFF (histogram fuzzy filter). Authors in [11] presented a PAFF (parametric adaptive fuzzy filter), which works effectively when the noise ratio is greater than $20 \%$. In [12], a rule-based method using local characteristics of the signal was presented which reduced Gaussian noise effect and preserved the edges. In [13], a hierarchical fuzzy approach was used to perform detail sharpening.

To the best of our knowledge, so far the fuzzy concepts have not been used in blur identification. In this paper, we present a novel algorithm using fuzzy sets and Radon 


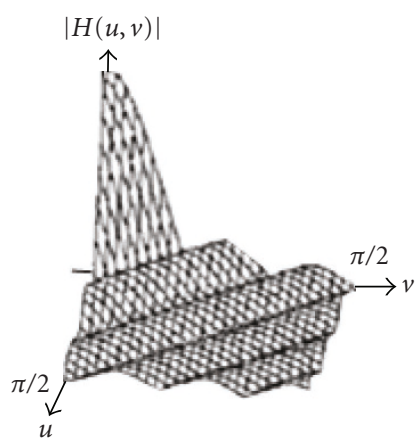

FIGURE 1: The frequency response of the uniform linear motion blur (a SINC shape function) with $L=7.5$ pixels, $\phi=\pi / 4$.

transform to find the motion blur parameters in presence or absence of additive noise. This new method improves our last works (presented in $[1,7]$ ) by supporting lower SNRs (i.e., an improvement between $3-5 \mathrm{~dB}$ ) and providing more precise answers.

We have implemented our method using Matlab 7 functions and tested it on 80 randomly selected standard images of $256 \times 256$ pixels. The accuracy of our method was evaluated by determining the mean and standard deviation of differences between actual and estimated angle/length parameters (in the following sections this difference is referred to as an error). In comparison with other methods listed above, our method supports lower SNRs. We measured the lowest allowed SNR in our method experimentally which was about $22 \mathrm{~dB}$ in average.

The rest of the paper is organized as follows. In Section 2, the motion blur parameters are introduced. Section 3 describes finding parameters in noise free images. The problem in noisy images and the use of fuzzy sets in motion length estimation are addressed in Section 4. Experimental results are provided in Section 5. In Section 6, we compare our method with other methods and finally we present the conclusion in Section 7.

\section{MOTION BLUR ATTRIBUTES}

The general form of the motion blur function is given as follows [7]:

$$
h(x, y)= \begin{cases}\frac{1}{L}, & \text { if } \sqrt{x^{2}+y^{2}} \leq \frac{L}{2}, \frac{x}{y}=-\tan (\phi), \\ 0, & \text { otherwise. }\end{cases}
$$

As seen in (2), motion blur function depends on two parameters: motion length $(L)$ and motion direction $(\phi)$. Figure 1 shows the frequency response of this function for $L=7.5$ pixels and $\phi=\pi / 4$.

The frequency response of $h$ is a SINC function. This implies that "if an image is affected only by motion blur and there is no additive noise, then we can see dominant parallel dark lines in its frequency response (Figure 2(b)) that correspond to very low near-zero values $[2,5,6,14,15]$." Figure 2 shows the lake image corrupted by motion blur with

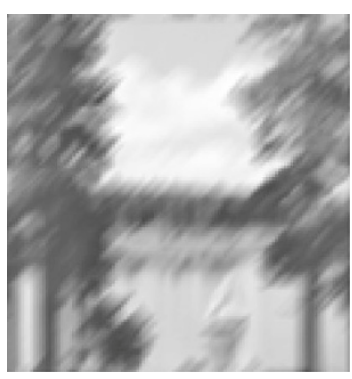

(a)

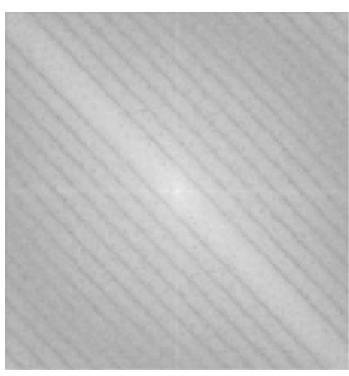

(b)
Figure 2: (a) The lake image degraded by linear motion blur using $L=20$ pixels, $\phi=45^{\circ}$, (b) Fourier spectrum of (a).

no additive noise and its Fourier spectrum, in which the parallel dark lines are obvious. These parallel dark lines and the SINC structure in the frequency response of the degradation function are the most critical data that are used in our method.

\section{MOTION BLUR PARAMETER ESTIMATION IN NOISE FREE IMAGES}

In this section, we propose a solution for cases in which the image is corrupted by a degradation function without additive noise (i.e., $n(x, y)=0$ ).

In the absence of noise, (3) concludes that

$$
G(u, v)=F(u, v) \cdot H(u, v),
$$

where $G(u, v), F(u, v)$, and $H(u, v)$ are frequency responses of the observed image, original image, and the degradation function, respectively. In this case, the motion blur parameters are determined as described in the following subsections.

\subsection{Motion direction estimation}

To find motion direction, we used the parallel dark lines that appear in the Fourier spectrum of a degraded image, an example of which is shown in Figure 2(b). In [1], we showed that the motion blur direction $(\phi)$ is equal to the angle $(\theta)$ between any of these parallel dark lines and the vertical axis. Therefore, to find motion direction, it is enough to find the direction of these parallel dark lines. However, we supposed $I=\log |G(u, v)|$ is a gray-scale image in spatial domain to which we can apply any line fitting method to find the direction of a line. Among many line fitting methods that were applicable, we used Radon transform [16] either in the form of (4) or (5):

$$
\begin{aligned}
& R(\rho, \theta)=\int_{-\infty}^{\infty} \int_{-\infty}^{\infty} g(x, y) \delta(\rho-x \cos \theta-y \sin \theta) d x d y, \\
& R(\rho, \theta)=\int_{-\infty}^{\infty} g(\rho \cos \theta-s \sin \theta, \rho \sin \theta+s \cos \theta) d s .
\end{aligned}
$$


The advantage of Radon transform to other line fitting algorithms, such as Hough transform and robust regression [17], is that one does not need to specify candidate points for the lines. To find direction of these lines, let $R$ be the Radon transform of an image, then the position of high spots along the $\theta$ axis of $R$ shows the direction [16]. Figure 4 shows the result of applying Radon transform to the log of Fourier transform of an image which was corrupted by a linear motion blur (direction $45^{\circ}$ ) with no additive noise. To find the high spots, we can use any peak detection algorithm like Cepstrum analysis.

More details of using Radon transform for finding motion direction are given in our previous work [7].

\subsection{Motion length estimation}

After finding motion direction, we rotated the coordinate system of $\log |G(u, v)|$, rather than rotating the observed image, to align it with motion direction. Rotating the coordinate system solves the problems that occur in image rotation such as interpolation and out of range pixels. Because of the rotation effect, some parts of Fourier spectrum will appear in areas out of the coordinate system support, as a result the same number of valid data will not be available in all columns in the new coordinate system. Most of valid data is located in the column passing through frequency center. The presented algorithm is based on the central peaks and valleys in the Fourier spectrum, therefore this rotation has no effect on precision and robustness of the algorithm.

In this case, the uniform motion blur equation is onedimensional like (6) [18]:

$$
h(i)= \begin{cases}\frac{1}{L} & \text { if }-\frac{L}{2} \leq i \leq \frac{L}{2}, \\ 0 & \text { otherwise. }\end{cases}
$$

The continuous Fourier transform of $h$, which is a SINC function is shown in (7) [19]:

$$
H_{c}(u)=\frac{2 \operatorname{Sin}(u \pi L / 2)}{u \pi L} .
$$

The discrete version of $H$ in horizontal direction is shown in $(8)[2,19]$ :

$$
H(u)=\frac{\operatorname{Sin}(L u \pi / N)}{L \operatorname{Sin}(u \pi / N)}, \quad 0 \leq u \leq N-1,
$$

where $N$ is the image size. To find $L$ we tried to solve the equation $H(u)=0$, (i.e., finding zero values of a SINC function). Solving this equation leads to solving (9):

$$
\begin{gathered}
\operatorname{Sin}\left(\frac{L u \pi}{N}\right)=0, \\
u=\frac{k \pi}{L W} \quad \text { such that } W=\frac{\pi}{N}, k>0 .
\end{gathered}
$$

If $u_{0}$ and $u_{1}$ are two successive zero points such that $H\left(u_{0}\right)=H\left(u_{1}\right)=0$, then

$$
u_{1}-u_{0}=\frac{N}{L}
$$

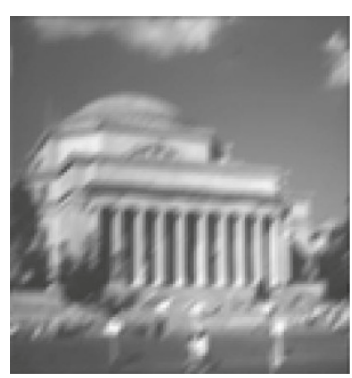

(a)

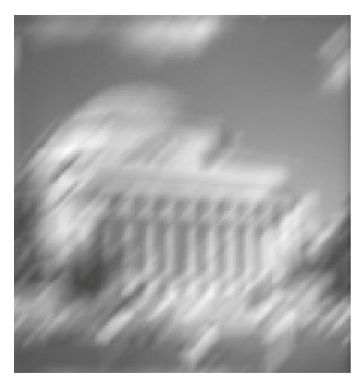

(c)

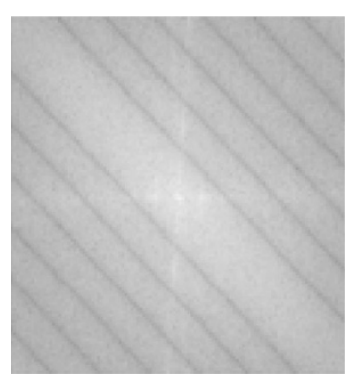

(b)

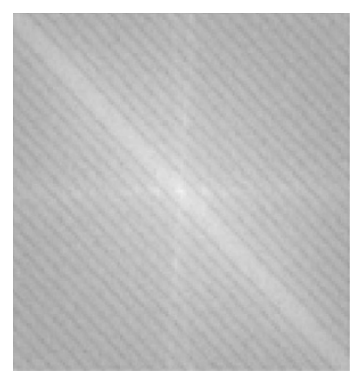

(d)
Figure 3: (a) A motion blurred image with $L=10$ pixels, $\phi=45^{\circ}$, (b) Fourier spectrum of (a), (c) motion blurred image with $L=30$ pixels, $\phi=45^{\circ}$, (d) Fourier spectrum of (c). The sizes of (a) and (c) are $256 \times 256$ pixels.

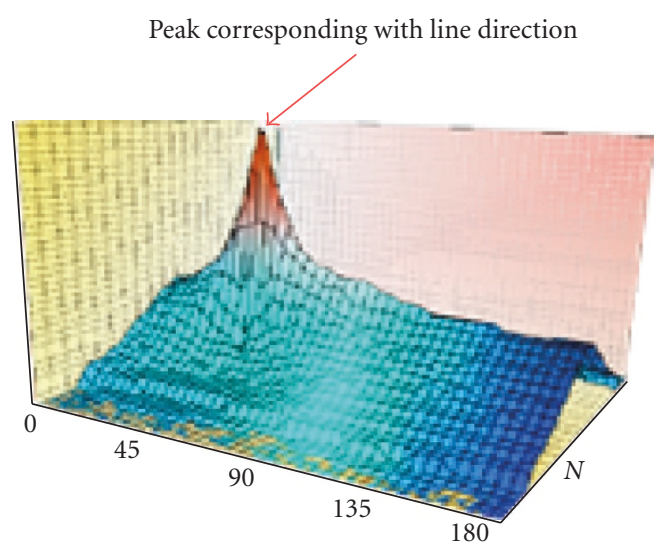

Figure 4: The result of applying Radon transform to the log of Fourier transform of an image that was degraded using linear motion blur with direction $45^{\circ}$ and no additive noise.

which results in

$$
L=\frac{N}{d},
$$

where $d$ is the distance between two successive dark lines in $\log (|G(u, v)|)$.

Figures 3(b) and 3(d) show visualizations of $\log (|G(u, v)|)$ for two motion blurred sample images. To find $d$ and use it to calculate $L$ using (12), we should find $u$ such that $G(u)$ is zero or near zero. Those points for which $G(u)$ is near 


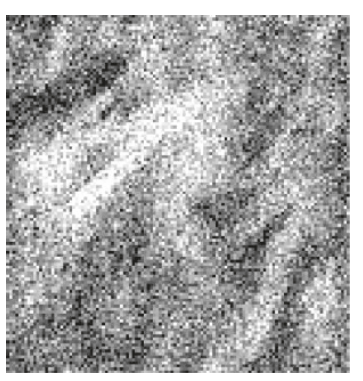

(a)

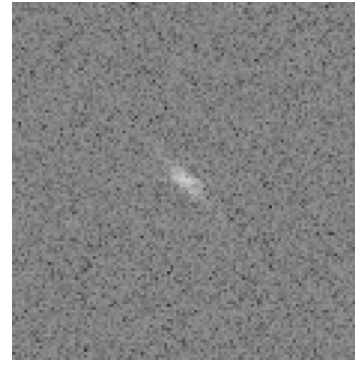

(b)
Figure 5: (a) The image $(256 \times 256)$ of Barbara which is degraded by motion blur with parameters $L=30$ pixels, $\phi=45^{\circ}$ and Gaussian additive noise with zero mean and variance $=0.04(\mathrm{SNR}=30 \mathrm{~dB})$. (b) its Fourier spectrum.

zero are categorized into two groups. The first group corresponds to the straight dark lines that are created by motion blur $(H(u)=0)$ and the second group is created by actual pixel values $(F(u)=0)$. To calculate $d$, we should use the first group of $u$. To do this robustly, we used fuzzy set as described in Section 4.2.

\section{MOTION BLUR PARAMETER ESTIMATION IN NOISY IMAGES}

When noise, usually with a Gaussian distribution, is added to a degraded image, the parallel dark lines in frequency response of degraded image become weak and some of them disappear. If noise variance increases, then more such dark lines disappear. Figure 5(a) shows Barbara image degraded by motion blur and additive noise, Figure 5(b) shows the frequency response of Figure 5(a). To overcome the noise effect, in the following sections we propose a novel, simple, and robust algorithm based on Radon transform and fuzzy sets to estimate motion direction and length, respectively.

\subsection{Motion direction estimation in noisy images}

The concepts we have used here is similar to the one used for noiseless images. Looking at Figure 5(b) we can see a white bound around the image center. This white bound is generated by the SINC structure of frequency response of motion blur function shown in Figure 1. The direction of white bound exactly matches with the direction of disappeared dark lines, so it also corresponds to the direction of motion blur. Therefore, to find motion blur direction, it is enough to find the direction of this white bound, consisting of several parallel white lines. Using Radon transform, we can find the direction $(\theta)$ of these white lines [7].

\subsection{Motion length estimation in noisy images using fuzzy sets}

In presence of noise, the parallel dark lines in frequency response of a degraded image become weak and some of them disappear. In low SNRs, these dark lines disappear completely. Equation (13) shows frequency domain version of (1)

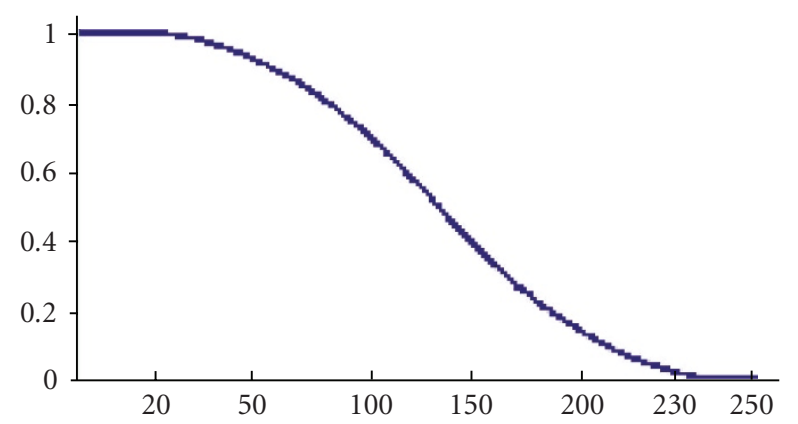

FIGURE 6: The $Z$-structure of membership function introduced by (15) when $a=20$ and $c=230$.

in the presence of noise. Here $W(u, v)$ has different parameters in Gaussian distribution compared to $w(x, y)$ :

$$
G(u, v)=H(u, v) \cdot F(u, v)+W(u, v)
$$

Since noise is a random parameter, its effect on pixels of a dark line is different. The question is which pixels belong to the disappeared dark lines. In $\log (|G(u, v)|)$, darker pixels are better candidates to be a part of a dark line than others. Which pixels are dark pixels? And can we certainly claim that the other pixels are not part of a dark line? Because of noise effects, we cannot answer these questions with certainty. This uncertainty leads us to use fuzzy concepts to find dark lines in frequency response of degraded images. In fact, each pixel in the frequency response of a degraded image can be a part of a dark line with different possibility, therefore, we define a fuzzy set for each row of $\log (|G(u, v)|)$ in rotated coordinate system as follows:

$$
A_{i}=\left\{\left(x, \mu_{n(x)}\right) \mid x \in(1, \ldots, N), n(x)=\log (|G(i, x)|)\right\},
$$

where $N$ is the number of columns in image and $i$ is the row number. We define the membership function $\mu_{u}$ as the $Z$ function, because the darker pixels are better candidates to belong to disappeared dark lines while lighter ones are worse candidates. The $z$-function models this property using the following equation:

$$
\mu_{u}= \begin{cases}1, & u \leq a, \\ 1-2 \times \frac{(u-a)^{2}}{(c-a)}, & a<u \leq \frac{(a+c)}{2}, \\ 2 \times \frac{(u-c)^{2}}{(c-a)}, & \frac{(a+c)}{2}<u \leq c, \\ 0, & \text { otherwise. }\end{cases}
$$

Figure 6 shows a plot of this function. In (15), $a$ and $c$ are two constant values that are specified heuristically. The best values that we found were $a=20$ and $c=230$ for a 256 level gray-scale image. We used the same values of $a$ and $c$ for all images. The columns of $\log (|G(u, v)|)$ with higher membership values in all sets $\left(A_{i}\right)$ are the best candidate for the dark 


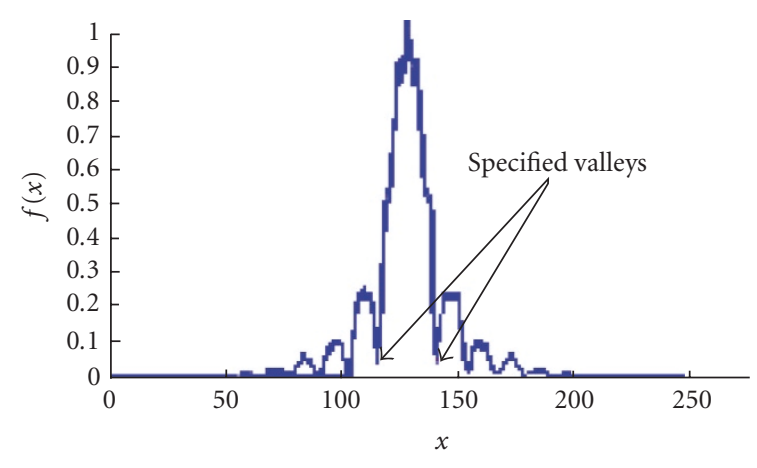

FIGURE 7: $f(x)$ of an image with no additive noise.

lines. Therefore, we used Zadeh $t$-norms [20] to find intersection of these sets:

$$
B=\left\{\left(x, \mu_{x}^{\prime}\right) \mid \mu_{x}^{\prime}=t\left(\mu_{1 x}, \ldots, \mu_{M x}\right), x \in(1, \ldots, N)\right\} .
$$

In this equation, $B$ is intersection of sets, $M$ is number of rows in $\log (|G(u, v)|), \mu_{i x}$ shows the membership value of $x$ in $A_{i}$, and $t$ is Zadeh $t$-norm. Now we define $f(x)$, the possibility that column $x$ does not belong to a dark line, as follows:

$$
f(x)= \begin{cases}1-\mu^{\prime}, & x \in B, \\ 0, & \text { otherwise. }\end{cases}
$$

Figure 7 shows the $f(x)$ that was obtained from a degraded image with $L=30$ pixels with no additive noise. Looking carefully at this figure, it is obvious that $f(x)$ has a SINC structure and valleys in $f(x)$ (valleys in the Fourier spectrum of degradation function) correspond to the dark lines.

Figure 8 shows $f(x)$ of an image corrupted by linear motion blur with $L=30$ pixels and added Gaussian noise with $\sigma_{w}^{2}=1$ and $\mathrm{SNR}=25 \mathrm{~dB}$.

All valleys of $f(x)$ are candidates of dark line places but some of them may be false. The best ones are valleys that correspond to SINC structure in Fourier spectrum of degradation function. These valleys are in two sides of the central peak as shown in Figures 7 and 8. By finding these valleys using a conventional pitch detection algorithm, their distance can be calculated. Because of the SINC structure, this distance is twice the distance between two successive parallel dark lines. Therefore, by using (12) we can find motion length using the following equation:

$$
L=\frac{2 \times N}{r},
$$

where $r$ is the distance between these valleys and $N$ is the image size. This equation is derived from (12), by setting $d=r / 2$, where $d$ is successive lines distance. It is important to note that the values of $f(x)$ are not the same in different images, while $f(x)$ consists of peaks and valleys which depend on degradation function but not on the image. The advantage of this algorithm is that it works in low SNR and its robustness does not depend on $L$ and $\phi$.

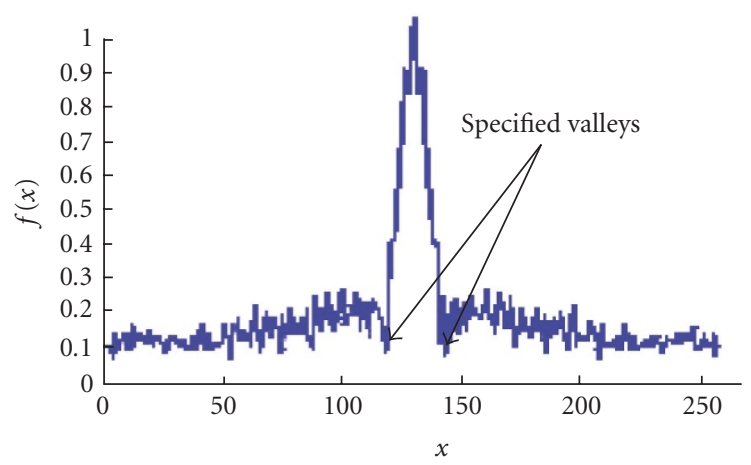

FIGURE 8: $f(x)$ of an image corrupted by linear motion blur with $L=30$ pixels and additive Gaussian noise $(\mathrm{SNR}=25 \mathrm{~dB})$.

\section{EXPERIMENTAL RESULTS}

We have applied the above algorithms on $80(256 \times 256)$ standard images such as Camera-man, Lena, Barbara, Baboon, that were degraded by different orientations and lengths of motion blur (i.e., $0^{\circ} \leq \phi \leq 180^{\circ}$ and $10 \leq L \leq 50$ pixels). Then we added Gaussian noise with zero mean and different variances $\left(0.01 \leq \sigma_{w}^{2} \leq 0.61\right)$ to these images. To create a blurred image, three random variables were produced as follows:

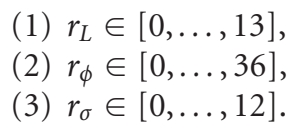

Then the blur parameters were calculated using the following equations:

$$
\begin{gathered}
L=r_{L} \times 3+10 ; \\
\phi=r_{\phi} \times 10 ; \\
\sigma_{w}^{2}=r_{\sigma} \times 0.05+0.01 .
\end{gathered}
$$

In each iteration, a degraded image was created using these parameters. Therefore, regarding intervals defined for $r_{L}, r_{\phi}$, $r_{\sigma}$, and (19), 14 different lengths, 37 different directions and 13 different Gaussian noise variances could be combined to create a sample set of degraded images. We selected $80 \mathrm{im}$ ages from this set to test our algorithm. Then we used our algorithm to find motion blur parameters of the blurred images created by the mentioned procedure. Cepstrum analysis, that is, a standard pitch detection algorithm was used to find valleys in $f(x)$. Additionally, the properties of SINC structure of $f(x)$ were used to discard false detected valleys and to increase the precision of the method. Using this customized Cepstrum analysis, the valleys around the central peak with the same distances were accepted. After finding motion blur parameters, Wiener filter was used to restore the original images.

Tables 1 and 2 show the summary of results. In these tables, the columns named "angle tolerance" and "length tolerance" show the absolute value of errors (the difference between the actual values of the angle and length and their estimated values), respectively. The low values of the mean and 
TABLE 1: Experimental results of our algorithm on 80 degraded standard images $(256 \times 256)$ with no additive noise.

\begin{tabular}{l|cc}
\hline Cases & $\begin{array}{c}\text { Angle tolerance } \\
\text { (degree) }\end{array}$ & $\begin{array}{c}\text { Length tolerance } \\
\text { (pixels) }\end{array}$ \\
\hline Best estimate & 0 & 0.0 \\
Worst estimate & 2 & 1.9 \\
Average estimate & 0.6 & 0.9 \\
Standard deviation & 0.7 & 0.4 \\
\hline
\end{tabular}

TABle 2: Experimental results of our algorithm on 80 degraded standard images $(256 \times 256)$ with additive noise with zero mean and 0.01 to 0.6 variance $(\mathrm{SNR}>22 \mathrm{~dB})$.

\begin{tabular}{l|cc}
\hline Cases & $\begin{array}{c}\text { Angle tolerance } \\
\text { (degree) }\end{array}$ & $\begin{array}{c}\text { Length tolerance } \\
\text { (pixels) }\end{array}$ \\
\hline Best estimate & 0 & 0.0 \\
Worst estimate & 2 & 2.5 \\
Average estimate & 0.9 & 0.9 \\
Standard deviation & 0.69 & 0.55 \\
\hline
\end{tabular}

standard deviation of errors show the high precision of our algorithm. The worst case of the algorithm in estimation of motion length occurred when $L>40$ pixels and its best case happened when $10 \leq L \leq 26$ pixels.

For estimating motion direction, there was no specific range for worst and best cases of the algorithm. These cases may occur in each direction.

If we define SNR as (20):

$$
\mathrm{SNR}=10 \log _{10}\left(\frac{\sigma_{f}^{2}}{\sigma_{w}^{2}}\right)
$$

where $\sigma_{f}^{2}$ denotes image variance and $\sigma_{w}^{2}$ defines noise variance, then our algorithm shows a robust behavior at SNR $>22 \mathrm{~dB}$. Decreasing SNR values increases algorithm estimation error. As an example for a specified image with $L=18$ pixels and $\mathrm{SNR}=15 \mathrm{~dB}$, the motion length estimation error was about 10 pixels. At SNR $=20 \mathrm{~dB}$, the estimation error was about 7 pixels for the same image. Figures 9 and 10 show noisy degraded images with low SNRs. Their motion blur parameters were estimated successfully by our algorithm. Also we studied the effect of changing the values of parameters $a$ and $c$ in (15) on the algorithm. The best values for these parameters were $a=20$ and $c=230$, that were calculated heuristically. Changing the values of $a$ and $c$ in the range of \pm 5 and \pm 10 , respectively, did not have significant effect on algorithm precision. But changing these parameters beyond these ranges decreases the precision of the algorithm.

\section{COMPARISON WITH RELATED METHODS}

A comparison with related methods shows that the method presented in this paper is more robust, has higher precision, and supports lower SNRs.

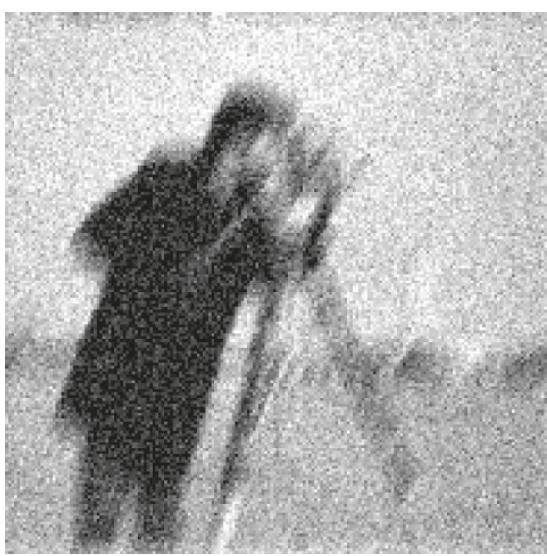

FIguRE 9: Camera picture which was degraded by $L=20$ pixels, $\phi=60^{\circ}$ and Gaussian additive noise $(\mathrm{SNR}=30 \mathrm{~dB})$. Estimated values for this image using our algorithm were $L=21.8$ pixels and $\phi=58.7^{\circ}$.

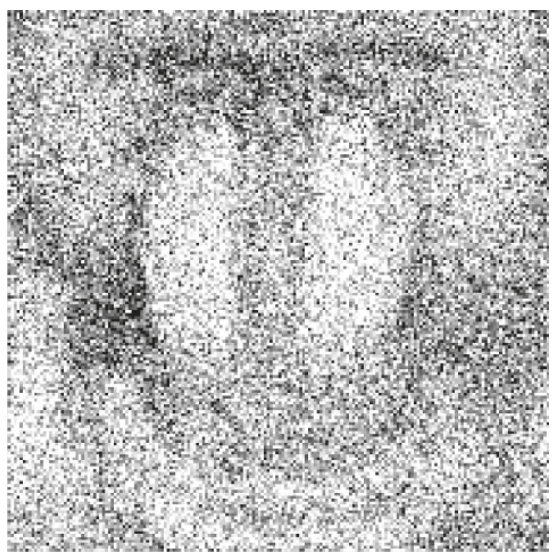

Figure 10: Baboon picture which was degraded by $L=10$ pixels, $\phi=135^{\circ}$ and Gaussian additive noise $(\mathrm{SNR}=25 \mathrm{~dB})$. Estimated values for this image using our algorithm were $L=8$ pixels and $\phi=136.8^{\circ}$.

In [2], the experimental results were presented briefly and there was no overall experimental results. Their algorithm was tested only on two degraded images in horizontal direction using $L=11$ pixels. As authors said, the estimated length for the first image with SNR $=40 \mathrm{~dB}$ was $L=11.1$ pixels which was the same as results for the second image with $\mathrm{SNR}=30 \mathrm{~dB}$. In addition, the authors presented a restored image with $\mathrm{SNR}=23.3 \mathrm{~dB}$, but they did not present parameter estimation for this image. The weak point of this method was that the authors presented results for only two images. To compare our algorithm with method presented in [2], we applied our algorithm to similar images with the same parameters, which resulted in estimation of $L=11.6$ pixels when $\mathrm{SNR}=40 \mathrm{~dB}$ and $L=11.8$ pixels when SNR $=30 \mathrm{~dB}$.

Authors in [21] did not discuss additive noise but they tried to solve the problem in noise free images. The average estimation error that they reported in noise free texture 
images was $3.0^{\circ}$ in direction and 4.1 pixels in length which were both worst than the ones found in our method under similar conditions.

In another paper [15] authors presented an estimation error of $1^{\circ}-3^{\circ}$ in direction and $4-6$ pixels in length for noise free images which are not as good as the ones obtained with our method.

The authors in [14] did not discuss the precision and SNR support of their method. It has also a limitation in motion length where $L<15$ pixels. Our method has no limitation in motion length in theory. We tested it using $L \leq 50$ pixels and the results were satisfactory.

The researchers in [6] did not show the lowest SNR that their method could support. They reported that their method worst case estimation error was about $5^{\circ}$ in direction and 2.5 pixels in length. These results were analyzed with noise variances of 1 and 25 .

In addition, the methods given in $[3,22]$ were valid for SNR as low as $40 \mathrm{~dB}$. The authors did not provide any information about the precision of their methods.

The algorithms presented in $[4,5]$ have no exhaustive experimental results.

In our latest work we provided a method that had a precise estimation of parameters in BSNR > $30 \mathrm{~dB}$ (which is about SNR > $25 \mathrm{~dB}$ [7]). Overall, our method supports lower SNR than other methods and it gives better precision in most cases.

\section{CONCLUSION}

In this paper we presented a robust method to estimate the motion blur parameters, namely, direction and length. Although fuzzy methods are used in many research areas, but to the best of our knowledge, their usage in blur identification have not been reported yet. We used fuzzy set concepts to find motion length. This is a novel idea in this field. We showed the robustness of this method for noisy and noiseless images. To estimate motion direction we used Radon transform. This helped us to overcome the difficulties with Hough transform and similar methods to find the candidate points for line fitting.

The main advantage of our algorithm is that it does not depend on the input image. To evaluate the performance of our method, we degraded 80 standard images with different values of motion direction and length. The motion blur parameters that were estimated by our method were compared with their initial values for each image. The comparison of the low value for mean and standard deviation of errors between the estimated values and the actual ones showed the high accuracy of our method.

We believe that the performance of motion blur parameter estimation algorithms can be improved if the noisy degraded images are processed with specific noise removal algorithms which are able to remove noises while preserving edges. After applying such noise removal methods we can implement our algorithm for motion blur parameter estimation to obtain better results. In future, we plan to extend our work to develop such noise removal methods.

\section{ACKNOWLEDGMENT}

We highly appreciate Iran Telecommunication Research Center for its financial support to this research, that is part of a Ph.D. thesis.

\section{REFERENCES}

[1] M. E. Moghaddam and M. Jamzad, "Finding point spread function of motion blur using radon transform and modeling the motion length," in Proceedings of the 4th IEEE International Symposium on Signal Processing and Information Technology (ISSPIT '04), pp. 314-317, Roma, Italy, December 2004.

[2] Q. Li and Y. Yoshida, "Parameter estimation and restoration for motion blurred images," IEICE Transactions on Fundamentals of Electronics, Communications and Computer Sciences, vol. E80-A, no. 8, pp. 1430-1437, 1997.

[3] M. M. Chang, A. M. Tekalp, and A. T. Erdem, "Blur identification using the bispectrum," IEEE Transactions on Acoustics, Speech, and Signal Processing, vol. 39, no. 10, pp. 2323-2325, 1991.

[4] M. Cannon, "Blind deconvolution of spatially invariant image blurs with phase," IEEE Transactions on Acoustics, Speech, and Signal Processing, vol. 24, no. 1, pp. 58-63, 1976.

[5] R. Bhaskar, J. Hite, and D. E. Pitts, "An iterative frequencydomain technique to reduce image degradation caused by lens defocus and linear motion blur," in IEEE Geoscience and Remote Sensing Symposium (IGARSS '94), vol. 4, pp. 2522-2524, Pasadena, Calif, USA, August 1994.

[6] C. Mayntz, T. Aach, and D. Kunz, "Blur identification using a spectral inertia tensor and spectral zeros," in IEEE International Conference on Image Processing (ICIP '99), vol. 2, pp. 885-889, Kobe, Japan, October 1999.

[7] M. E. Moghaddam and M. Jamzad, "Blur identification in noisy images using radon transform and power spectrum modeling," in Proceedings of the 12th IEEE International Workshop on Systems, Signals and Image Processing (IWSSIP '05), pp. 347-352, Chalkida, Greece, September 2005.

[8] K.-H. Yap and L. Guan, "A fuzzy blur algorithm to adaptive blind image deconvolution," in Proceedings of the 7th IEEE International Conference on Control, Automation, Robotics and Vision (ICARCV'02), pp. 34-38, Singapore, Republic of Singapore, December 2002.

[9] J.-H. Wang, W.-J. Liu, and L.-D. Lin, "Histogram-based fuzzy filter for image restoration," IEEE Transactions on Systems, Man, and Cybernetics_Part B: Cybernetics, vol. 32, no. 2, pp. 230-238, 2002.

[10] J.-H. Wang and M.-D. Yu, "Image restoration by adaptive fuzzy optimal filter," in Proceedings of the IEEE International Conference on Systems, Man and Cybernetics, vol. 1, pp. 845848, Vancouver, BC, Canada, October 1995.

[11] J.-H. Wang and L.-D. Lin, "Image Restoration using parametric adaptive fuzzy filter," in IEEE Conference of the North American Fuzzy Information Processing Society (NAFIPS '98), Pensacola, Fla, USA, August 1998.

[12] K. Arakawa, "Fuzzy rule-based signal processing and its application to image restoration," IEEE Journal on Selected Areas in Communications, vol. 12, no. 9, pp. 1495-1502, 1994.

[13] S. Suthaharan, Z. Zhang, and A. Harvey, "FFF: fast fuzzy filtering in image restoration," in IEEE Region 10 Annual Conference Speech and Image Technologies for Computing and Telecommunications (TENCON '97), vol. 1, pp. 9-12, Brisbane, Australia, December 1997. 
[14] Y. Li and W. Zhu, "Restoration of the image degraded by linear motion," in Proceedings of the 10th IEEE International Conference on Pattern Recognition (ICPR '90), vol. 2, pp. 147-152, Atlantic City, NJ, USA, June 1990.

[15] I. M. Rekleitis, "Optical flow recognition from the power spectrum of a single blurred image," in IEEE International Conference on Image Processing (ICIP '96), vol. 3, pp. 791-794, Lausanne, Switzerland, September 1996.

[16] P. Toft, The radon transform-theory and implementation, Ph.D. thesis, Department of Mathematical Modeling, Technical University of Denmark, Copenhagen, Denmark, June 1996.

[17] R. C. Gonzalez and R. E. Woods, Digital Image Processing, Prentice Hall, Upper Saddle River, NJ, USA, 2nd edition, 2002.

[18] M. R. Banham and A. K. Katsaggelos, "Digital image restoration," IEEE Signal Processing Magazine, vol. 14, no. 2, pp. 2441, 1997.

[19] A. V. Oppenheim, A. S. Willsky, and S. H. Nawab, Signals and Systems, Prentice Hall, Upper Saddle River, NJ, USA, 2nd edition, 1996.

[20] H. J. Zimmermann, Fuzzy Set Theory, Kluwer Academic, Dordrecht, The Netherlands, 1996.

[21] I. M. Rekleities, "Steerable filters and cepstral analysis for optical flow calculation from a single blurred image," in Proceedings of the Vision Interface Conference, pp. 159-166, Toronto, Ontario, Canada, May 1996.

[22] A. T. Erdem and A. M. Tekalp, "Blur identification based on bispectrum," in Proceedings of the European Signal Processing Conference (EUSIPCO '90), Barcelona, Spain, September 1990.

Mohsen Ebrahimi Moghaddam received his M.S. degree in software engineering from Sharif University of Technology, Tehran, Iran. Currently, he is a Ph.D. Candidate in the Department of Computer Engineering, Sharif University. The present work is the main core of his Ph.D. thesis. His main research interests are image processing, machine vision, data structures, and algorithm design.

Mansour Jamzad has obtained his M.S. degree in computer science from McGill University, Montreal, Canada and his Ph.D. degree in electrical engineering from Waseda university, Tokyo, Japan. For a period of two years after graduation he worked as a Post Doctorate Researcher in the Department of Electronics and Communication Engineering, Waseda University. He became an Assistant Professor at the Department of

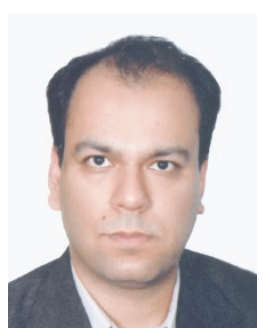
Computer Engineering, Sharif University of Technology, Tehran, Iran since 1995 . He has been teaching digital image processing and machine vision graduate courses in the last 10 years. He is a Member of IEEE and his main research interests are digital image processing, machine vision and its applications in industry, robot vision, and fuzzy systems. 